\title{
Epidemiology of kidney cancer in Turkistan region
}

\author{
Assel Zhumagulova ${ }^{1,2}$, Ruslan Nurbaba ${ }^{3}$ \\ ${ }^{I}$ Medicine Department, Hodge Ahmed Yasavi International Kazakh-Turkish University, Turkestan, Republic of Kazakhstan \\ ${ }^{2}$ City cancer center, Shymkent, Republic of Kazakhstan \\ ${ }^{3}$ South Kazakhstan Pharmaceutical Academy, Shymkent, Republic of Kazakhstan
}

Received: 2020-08-04.

Accepted: 2020-09-14

This work is licensed under a Creative Commons Attribution 4.0 International License
J Clin Med Kaz 2020; 6(60):101-102

Corresponding author:

Assel Zhumagulova.

E-mail: assel_skr@mail.ru;

ORCID:

\begin{abstract}
Introduction: The article presents epidemiological data on the cases of kidney cancer in Turkistan region depending on age and sex.

Purpose: Assess the incidence and neglect of kidney cancer in the Turkestan region.

Material and methods: Mathematical and statistical methods recommended by the Ministry of Healthcare were used.

Results: In 2013-2018 South Kazakhstan region there is a incidence of kidney cancer among men-4.4, among women-3.2 respectively. In addition, among cancer, kidney cancer is 8 among men and 12 among women. The maximum incidence peak is $50-59$ years. This means that the prevalence of the disease among the working-age population.

Conclusion: Thus, conducting a statistical analysis of the oncological state in the Turkestan region, we note: an increase in the incidence of kidney cancer, as well as a comparative increase in mortality, and finally an increase in the five-year survival rate. However, the increase in the number of patients first identified at stage IV proves that the level of oncological vigilance is still low.
\end{abstract}

Key words: disease, kidney cancer, Turkestan region, epidemiology

\section{Introduction}

Despite the fact that until recently the incidence of kidney cancer is rare among oncological diseases, there is now a marked trend of its growth in the world [1]. According to WHO, kidney cancer ranks the10th position among malignant neoplasms.

Every year there are 189.1 thousand new cases of kidney cancer in the world, 90,1 thousand of which are mortality. In Kazakhstan, the incidence of kidney cancer shows: in 2018 there were 1201 new cases of kidney cancer in the country, and in 2017 there were 1163 cases, the growth trend is 0.6 . However, mortality was noted in 341 cases (2018). For note, 300,000 kidney cancers are recorded annually in the United States, and 12,000 people dying from the disease each year. Kidney cancer is twice common among men than among women $[1,2]$.

The incidence of kidney cancer has a marked trend towards territorial variability (per 100,000 inhabitants) in Japan 1.5, cases, and in the Czech Republic there are 22 cases among men and 11 cases among women: this means dependence of kidney cancer on environment circumstances of people [3]. This pattern is maintained in Kazakhstan: in Karaganda and East Kazakhstan in 2017, 140 and 138 cases of kidney cancer were identified for the first time, respectively, and 68 new cases were identified in Turkistan region $[5,6]$.

The geographical prevalence of kidney cancer is different in specific differences between men and women, and the dependence of disease factors on different hypotheses proves the relevance of local/territorial epidemiological monitoring. The data used serve as a basis for improving cancer care [4].

\section{Material and methods}

The aim of the study is to determine the pattern of kidney cancer in the period from 2013-2018 and to forecast this disease for the period up to 2023 .

Materials and methods of research:

- The information basis of this work is the factors on patients first identified with morbidity in the territory of the Turkistan region, taking into account the age and sex of the inhabitants of the province, official statistics of KazNICR; 2018);

- Reports of the registry office for 5 years (2013-

- Health organization report on kidney cancer;

- CROP system documentation;

-Dynamic results of clinical outcomes based on outpatient maps $[5,6]$.

The study is based on indicators of different ages 
of patients, as well as selected indicators based on the world standard per 100 thousand population (\%). An asymmetric interval was used to estimate the difference among individual indicators (using Poisson's method) [7].

Methods recommended by the Ministry of healthcare of the Republic of Kazakhstan and other relevant methods of mathematical statistics recommended in epidemiological studies were used. During the study of analytical indicators of trends, the incidence was estimated by the method of least squares according to the methodology developed by the research Institute of Oncology. The statistical significance of the results obtained is assumed to be at least $\mathrm{P}<0.01[8,9]$.

Results and analysis: in 2017, 1163 new cases of kidney cancer were registered in the Republic of Kazakhstan, for this year 6.5 per 100 thousand inhabitants and the growth rate is 0.6. Malignant tumor among men is $4,4 \%$, takes the 8 th place (absolute number of 630 people), among women $3 \%$, respectively, takes the 12th place (absolute number of 533). In the Turkestan region in 2017, 82 kidney neoplasms were registered, the incidence per 100 thousand inhabitants is 2.8 , 'extraordinary' growth dynamics of kidney cancer from 2013 to 2017 was 2.0. The increase in the Turkestan region is 1.2 , and in Kazakhstan it is 1.8. The annual average growth in the Turkestan region is 0.4 , in the Republic of Kazakhstan is 1.2 [5, 6].

In Turkestan region in 2013, among patients diagnosed with kidney cancer for the first time, $72.2 \%$ were registered at stage I and II, $15.6 \%$ at stage III, and $12.2 \%$ at stage IV. And in 2017 , at stage I and II-52.6\%, at stage III- $24.2 \%$, at stage IV$34.2 \%[5,6]$.

Analyzing the incidence of kidney cancer in the Turkestan region in 2013-2017, we can note an increase in the number of patients with kidney cancer registered for the first time at stages III and IV, as well as a decrease in the number of patients at stages I and II of the disease.

These statistics in the Turkestan region require improvement of screening programs for early detection of tumors and significant improvement of preventive work in the oncological community.

The growth of indicators of morphologically proven kidney cancer, in $201371.1 \%$, in $2017-77.9 \%$ in the Turkestan region, and in the Republic of Kazakhstan 78.6\% and $83.6 \%$, respectively, is associated with the implementation of modern research methods into the health care system [6].

\section{Results}

Mortality due to malignant tumors is one of the most important indicators. The annual mortality rate indicates a decrease in the rate of growth of morbidity among the population in the Turkestan region. If in 2013 it was $21.4 \%$, in 2017 it was $18.3 \%$. In Kazakhstan in 2017, this indicator per 100 thousand inhabitants was 2.0, the absolute number of 366 , in the Republic, the death rate from kidney cancer took the 14th place. 'Extraordinary' death rate per 100,000 inhabitants of the Turkestan region was 1.1 in 2013, and 1.3 in 2017.

The five-year survival rate from kidney cancer in the Turkestan region among residents in 2013 was $37.2 \%$ (with an absolute number of 142 people), and in $201746.3 \%$ (an absolute number of 244 people). This demonstrates an increase in the five-year survival rate from the disease.

\section{Conclusion}

Thus, conducting a statistical analysis of the oncological state in the Turkestan region, we note: an increase in the incidence of kidney cancer, as well as a comparative increase in mortality, and finally an increase in the five-year survival rate. However, the increase in the number of patients first identified at stage IV proves that the level of oncological vigilance is still low.

These facts require that the regional oncological and urological service timely / early detection of renal cell tumors and the use of modern methods of treatment, professional development of treating doctors in general, and in particular oncological vigilance.

Disclosures: There is no conflict of interest for all authors.

Acknowledgements: None.

Funding: None.

\section{References}

1. Davydov M. I., Axel E. M. Statistics of malignant neoplasms in Russia and CIS countries in 2009 [in Russian]. Bulletin of the national research Center named after N. N. Blokhin RAMS. 2011; 22(85):34-36.

2. Imyanitov E. N. Epidemiology and biology of kidney tumors [in Russian]. Practical Oncology. 2005; 3(6):137-140.

3. Information Bulletin on the state of cancer care in the Tyumen region for 2016. Tyumen. 2017; 52.

4. A.D. Kaprin, V. V. Starinsky, G. V. Petrova. Malignant neoplasms in Russia in 2016 [in Russian]. Moscow: fsbi "mnioi im. P. A. Herzen". 2017; 251.

5. Indicators of the cancer service of the Republic of Kazakhstan for 2018 (statistical and analytical materials). KAZ Nyior. Almaty. 2019.

6. Indicators of the cancer service of the Republic of Kazakhstan for 2016 (statistical and analytical materials). KAZ Nyior. Almaty. 2017.

7. Pasechnik D. G. Cytological features of kidney cancer [in Russian]. Urology. 2003; 5:58-62.

8. Petrosyan A. S. Oncourological pathology in the South of the Tyumen region [in Russian]. Academic journal of Western Siberia. 2013; 9(4):58-59.

9. Martel C. L., Lara P. N. Renal cell carcinoma: Status and future direction [in Russian]. Crit. Rev. Oncol. Hematol. 2003; 45:177-190. 